\title{
A Three-Mode Expressive Feature Model of Action Effort*
}

\author{
James W. Davis Hui Gao Vignesh S. Kannappan \\ Dept. of Computer and Information Science \\ Ohio State University \\ Columbus, OH 43210 USA \\ \{jwdavis, gaoh, kannappa\}@cis.ohio-state.edu
}

\begin{abstract}
We present an expressive feature model for recognizing the performance effort of human actions. A set of low and high effort examples for an action are initially factored into its three-mode principal components, followed by a learning phase to compute the expressive features required to bring the model estimation of effort into agreement with perceptual judgements. The approach is demonstrated using real and illusory movements.
\end{abstract}

\section{Introduction}

"Dynamic movements" are categories of movements performed under varying degrees of effort. Does his walk appear leisurely, or is he walking in a hurry? Does that package look heavy or light for her to carry? People are quite adept at identifying the amount of effort exerted by a person from subtle visual cues. For example, nuances in body motion can reveal how light or heavy a box is for a person to lift [12]. Our goal is to develop a computational system capable of identifying the "perceptual dynamics" associated with different performance efforts for the task of recognition.

Intelligent machines having a perceptual model of human action efforts are relevant to several domains and applications. Automatic video annotation of pedestrian walking pace and carrying load would help to enhance the current capability of surveillance systems. An ergonomic monitoring system able to detect improper lifting techniques (for heavy objects) may help reduce the occurrence of back injury and could be used to train patients with recurring injuries. Similarly, visual monitoring of athletic training could help prevent costly sports injuries by recognizing the onset of fatigue (characteristic changes in effort) during en-

\footnotetext{
* Appears in IEEE Workshop on Motion and Video Computing, Orlando, Florida, December 5-6, 2002, pp. 139-144.
}

durance workouts. A computer model of performance efforts could also be used to "warp" motion-capture animations into new efforts, and could potentially be used for searching digital motion libraries to find movements exhibiting a similar (or different) effort to the query example.

Instead of matching new movements to multitudes of training examples based on their proximity in some feature space, we present a system that learns the "expressive" motion features associated with a dynamic movement and computes a metric estimation of the effort. Our approach first constructs a three-mode factorization of low and high effort examples and then tunes the model using expressive features derived from additional labeled effort examples. A weighted-SSE minimization technique using perceptuallylabeled training data is employed to approximate human judgements of effort similarity. We demonstrate the approach with the task of recognizing the amount of effort exerted by a person carrying objects of different weight. Two movement illusions are also employed to further demonstrate the perceptual similarity of the model.

\section{Related Work}

There has been much recent work in computer vision on the detection, tracking, and recognition of human actions (See reviews $[1,6]$ ). Closely related to the recognition of action effort is analysis of style. A bilinear model was used in [13] for separating perceptual content and style parameters. A Fourier-based approach to generate human motion with emotional properties was described in [16]. A Hidden Markov Model (HMM) with entropy minimization was used by [3] to generate different state-based animation styles, and a Parameterized-HMM was used by [19] to model stylistic gesture variations. A factorization of motion capture data for extracting person-specific motion signatures was described in [18], and a movement exaggeration model using measurements of observability and predictability of joint angles was presented in [5].

The most related research addressing a three-mode anal- 
ysis of human movements over various performance efforts is presented in [9]. Arm segment velocities of 12 athletes throwing three differently weighted balls were examined using a three-mode factorization. The components themselves were manually inspected in an attempt to determine loadings signifying horizontal/vertical velocities, proximal/distal velocities, various throwing phases, and different skill levels of the throwers.

\section{Expressive Features as Key Features}

The success of perception (machine or man) relies on the ability to construct model representations whose assumptions and constraints reflect the structure and regularity of the world. A "key feature" is a property that can be reliably inferred in a particular context, where the likelihood of correctly indicating the property is high (few false targets) and the property itself has a significant prior probability of occurring [10].

With respect to recognizing action effort, do some properties of the movement vary consistently across effort to enable reliable discrimination of effort (e.g., which joint motions contribute the most to the overall percept of the action effort)? We refer to these key features as expressive features $^{1}$. We will show that certain trajectories can be used to match perceptual rankings of motions and therefore act as key features. We will also present a model that can infer these expressive features to enable the model to make judgements similar to those of people.

\section{Three-Mode Expressive Model}

Many times it is preferable to reduce the dimensionality of large data sets for ease of analysis (or recognition) by describing the data as linear combinations of a smaller number of latent, or hidden, prototypes. Singular value decomposition and principal components analysis are standard methods for achieving this data reduction, and have been successfully applied to several two-mode problems in computer vision (e.g., $[15,8,2])$. Three-mode factorization [14] is an extension of these traditional two-mode methods and offers a framework suitable to incorporating expressive features for efficient recognition of action efforts in a lowdimensional space.

Dynamic movements are inherently three mode: body pose (mode 1), time (mode 2), and effort (mode 3 ). The data can be organized into a cube $Z$ (See Fig. 1.a) with the rows in each frontal plane $Z_{k}$ comprising all the trajectories for a motion at a particular effort index $k$. This data cube could be "flattened", or rasterized, into an ordinary two-mode matrix, but this simply ignores the underlying

\footnotetext{
${ }^{1}$ An alternate meaning of expressive features is given by [4].
}

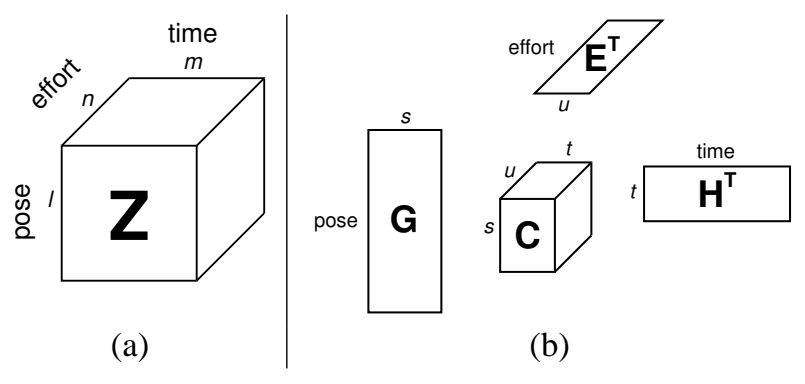

\section{Figure 1. (a) Three-mode configuration of dynamic-effort movement data. (b) Three- mode factorization of data.}

three-mode nature of the data. As is required with any principal components model, the data must first be normalized to a fixed length/size. We present results in the experimental section of this paper with automatic temporal segmentation and time-normalization of periodic carrying actions.

Three-mode factorization decomposes the data cube $Z$ into three orthonormal matrices $G, H$, and $E$ that span the column (pose), row (time), and slice (effort) spaces (See Fig. 1.b). Typically, each mode needs only to retain its first few components to capture most of the fit to $Z$. Note that any two of the three basis sets cannot be produced within a single two-mode factorization. The core matrix $C$ has three dimensions and represents the relationships of the components in $G, H$, and $E$ for reconstructing the original data in $Z$. An alternating least-squares algorithm for solving the three-mode factorization is presented in [7]. A related tensor decomposition can be found in [17].

The three-mode factorization of $Z$ can be concisely written in matrix form as

$$
Z=G C\left(E^{T} \otimes H^{T}\right)
$$

where $\otimes$ is the Kronecker product. Any frontal plane $Z_{k}$ for a given effort index $k$ (an action at a particular effort) can be formulated as

$$
Z_{k}=G\left(\sum_{r=1}^{u} e_{k r} C_{r}\right) H^{T}
$$

Therefore, we can reconstruct any frontal plane $Z_{k}$ by choosing the correct $e_{k r}$ component loadings from the effort mode $E$. For an unknown action (assumed to belong to the category), its effort values must be estimated.

\subsection{Estimating Action Effort}

The three-mode factorization for each data element $z_{i j k}$ of $Z$ can be written as a summation of three-mode elements, 
where the effort loadings can be isolated from the remaining factorized terms

$$
\begin{aligned}
z_{i j k} & =\sum_{p=1}^{s} \sum_{q=1}^{t} \sum_{r=1}^{u} g_{i p} h_{j q} e_{k r} c_{p q r} \\
& =\sum_{r=1}^{u} e_{k r}\left(\sum_{p=1}^{s} \sum_{q=1}^{t} g_{i p} h_{j q} c_{p q r}\right) \\
& =\sum_{r=1}^{u} e_{k r} \alpha_{i j r}
\end{aligned}
$$

If we have a nearly diagonal core (with $c_{p q r} \approx 0$ when $p \neq q$ ), we can further reduce the computations with $\alpha_{i j r}=\sum_{p=1}^{\min (s, t)} g_{i p} h_{j p} c_{p p r}$. The $e_{k r}$ values in Eqn. 5 can be estimated using least-squares methods.

Human movement exhibits smooth and predictable regularity with changes in the dynamic condition. Therefore only a few examples captured at distinct dynamic efforts may be all that is required to successfully model the actions. If we consider only two extreme efforts for an action (e.g., slow/fast walking, light/heavy lifting), the three-mode factorization of $Z$ (after mean-subtraction along the effort dimension) is reduced to contain a single effort parameter $e$. Movements examined between these extreme efforts should not deviate considerably from this three-mode basis.

To estimate the effort value for a movement of unknown effort with this reduced model, we can solve an error function $\mathcal{F}$ using the sum-of-squares of the input and its formulated reconstruction

$$
\mathcal{F}=\sum_{i} \sum_{j}\left(z_{i j}-e \cdot \alpha_{i j}\right)^{2}
$$

As all trajectories may not equally discriminate the action effort (all may not be expressive features), we augment the error function with expressibility weights $\mathcal{E}_{i}$ for each of the feature- $i$ trajectories

$$
\mathcal{F}^{\prime}=\sum_{i} \mathcal{E}_{i} \sum_{j}\left(z_{i j}-e \cdot \alpha_{i j}\right)^{2}
$$

For minimizing $\mathcal{F}^{\prime}$ to estimate the target effort parameter, we compute the derivative with respect to $e$ and re-arrange to produce

$$
\hat{e}=\frac{\sum_{i} \mathcal{E}_{i} \sum_{j} z_{i j} \alpha_{i j}}{\sum_{i} \mathcal{E}_{i} \sum_{j} \alpha_{i j}^{2}}
$$

Setting the $\mathcal{E}_{i}$ values to 1 in Eqn. 8 yields the standard leastsquares estimation of $e$. Non-uniform expressive feature weights in Eqn. 8 can be used to compute effort values that do not necessarily correspond to a standard minimization of SSE (as will be demonstrated).

\subsection{Learning Expressive Features}

To learn the expressive feature weights $\mathcal{E}_{i}$, we construct a second error function $J$ comparing additional training examples with known $e$ values (method to attain the $e$ values will be described in Sect. 5.4) to the estimated $\hat{e}$ values computed with Eqn. 8.

For a set of $k$ training motions $\tilde{Z}_{k}$ and their efforts $\tilde{e}_{k}$, we define the matching error as

$$
\begin{aligned}
J & =\sum_{k}\left(\tilde{e}_{k}-\hat{e}_{k}\right)^{2} \\
& =\sum_{k}\left(\tilde{e}_{k}-\frac{\sum_{i} \mathcal{E}_{i} \sum_{j} \tilde{z}_{i j k} \alpha_{i j}}{\sum_{i} \mathcal{E}_{i} \sum_{j} \alpha_{i j}^{2}}\right)^{2} \\
& =\sum_{k}\left(\tilde{e}_{k}-\frac{\sum_{i} \mathcal{E}_{i} B_{i j k}}{\sum_{i} \mathcal{E}_{i} A_{i j}}\right)^{2}
\end{aligned}
$$

This non-linear arrangement of the $\mathcal{E}_{i}$ values can be solved using a fast iterative gradient descent algorithm of the form

$$
\mathcal{E}_{i}(n+1)=\mathcal{E}_{i}(n)-\eta(n) \cdot \frac{\partial J}{\partial \mathcal{E}_{i}}
$$

with the gradients $\frac{\partial J}{\partial \mathcal{E}_{i}}$ computed over all $k$ training examples

$$
\begin{aligned}
\frac{\partial J}{\partial \mathcal{E}_{i}}= & 2 \sum_{k}\left(\tilde{e}_{k}-\frac{\sum_{i} \mathcal{E}_{i} B_{i j k}}{\sum_{i} \mathcal{E}_{i} A_{i j}}\right) \\
& \cdot \frac{A_{i j} \sum_{i} \mathcal{E}_{i} B_{i j k}-B_{i j k} \sum_{i} \mathcal{E}_{i} A_{i j}}{\left(\sum_{i} \mathcal{E}_{i} A_{i j}\right)^{2}}
\end{aligned}
$$

The learning rate $\eta$ is re-computed at each iteration to yield the best incremental update. A random-restart approach is employed to handle local minima. Following convergence of Eqn. 12, effort values can be estimated for new movements using Eqn. 8.

\section{Experiments}

We analyzed our approach in the context of determining the "carrying effort" of a person holding a bag (in one hand) of increasing weight while walking on a treadmill.

Motion data of the movements were collected, and the lightest and heaviest carry were used to construct the threemode basis. People were asked to compare each of the carrying movements to a set of synthetic movements and choose the best match (mimicking the computer recognition process). Given these perceptual mappings, the expressive feature weights were automatically learned to tune the three-mode model to produce effort values similar to the human judgements. Two illusory movements were additionally tested with the learned model and compared to human observations. 


\subsection{Motion Capture}

A Vicon-8 motion capture system with 14 video cameras was used to create a hierarchical skeleton of the body with 3-D joint-angle trajectories sampled at $30 \mathrm{~Hz}$ (Acclaim ASF/AMC format). The trajectories for the movements were lowpass filtered using a 5th order, zero-phase forward-and-reverse Butterworth filter with cut-off at $6 \mathrm{~Hz}$. Two walk cycles were automatically extracted from each sequence using trajectory curvature peaks, averaged into a single walk cycle, and time-normalized to 50 frames using spline interpolation. The joint positions as seen from a camera placed between a front and side view of the person (45 degrees) were computed and rendered. Recent video tracking advances (e.g., [11]) will be investigated in future work to extract these joint positions automatically (the focus in this paper is movement representations for recognition).

We captured 9 carrying sequences (carrying $0-40 \mathrm{lbs}$, in $5 \mathrm{lb}$ increments) of a person walking on a treadmill at 1.4 MPH. The lowest (lightest) and highest (heaviest) carrying efforts are shown in Fig. 2.a. Additionally, we produced 22 "synthetic" movements by linearly interpolating and extrapolating a three-mode model created from the position data for the lightest and heaviest carry (producing 2 lighter, 15 interpolated, and 5 heavier). These synthetic movements will be used in the perceptual mapping task.

\subsection{Input Representation}

At this point we must decide on a representation for the movements to construct the three-mode basis for recognition. Rather than committing to any particular set of higherlevel composite feature definitions, we represent movements more generally as sets of low-level motion trajectories. Figure 2.b compares the results of a standard leastsquares effort estimation with three-mode models for the carry examples using $x-y$ joint positions and 2-D jointangles. As both methods produce essentially the same results, we selected the 2-D angle representation as it has fewer degrees-of-freedom (10) and more invariants (e.g., translation, scale, and possibly rotation).

\subsection{Illusory Movements}

We additionally created an artificial low-effort carry $\check{Z}_{\text {low }}$ and high-effort carry $\check{Z}_{\text {high }}$ from manually altering the motion capture data. These two movements, when compared to the 22 synthetic model movements using a nonexpressive effort estimation (Eqn. 8 with all $\mathcal{E}_{i}=1$ ), both map to the same synthetic movement (\#11 of 22). However, these artificial movements perceptually appear quite distinctly as light and heavy efforts: $\check{Z}_{\text {low }} \rightarrow$ LOW-EFFORT and $\breve{Z}_{\text {high }} \rightarrow$ HIGH-EFFORT. These illusions will be used

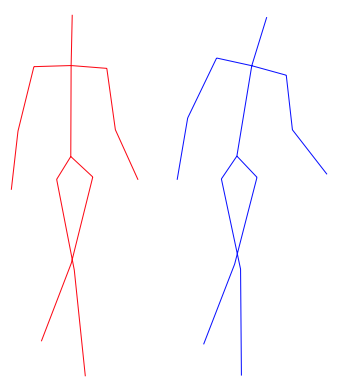

(a)

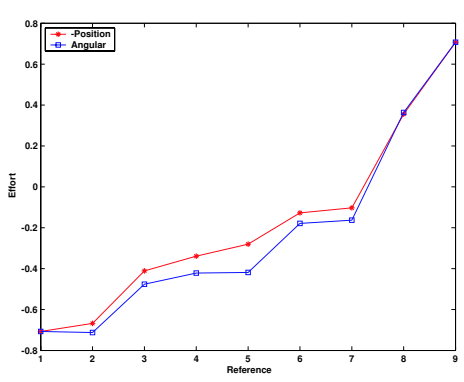

(b)

\section{Figure 2. (a) Light (left) and heavy (right) carry figures. A bag is held in the right hand. (b) Position vs. angular effort estimation.}

to further demonstrate the perceptually-based behavior of the model that was trained using only the real motions.

\subsection{Perceptual Mapping Task}

Nine people were given the task of matching the carrying movements to the set of synthetic model motions to provide a mapping for Eqn. 12 to learn the expressive features. Each person was capable of distinguishing side-by-side examples of extreme light and heavy carrying movements prior to the matching task.

A computer program was implemented to conduct the mapping task, and is shown in Fig. 3. On the left, one of 11 carry movies ( 9 real, 2 illusory) is shown as a "reference" movie. On the right, one of the 22 synthetic model movies is shown. Below the synthetic movie, a slider bar is provided for the user to quickly and easily seek through the possible synthetics. Moving the slider bar to the left or right instantly displays lighter or heavier looking movies, respectively. The reference and synthetic movies are synchronized and played repeatedly. The reference movies are selected in random order for each trial. The slider bar is initially set to a random position for each reference movie.

Once the user has made a choice of which synthetic movie most closely resembles the reference movie, the person selects a confirmation box and clicks the NEXT button to load the next reference movie. The program records the synthetic movie choice for each reference movie. Two complete trials were required, with the first trial used only to familiarize the person with the program and the movies.

\subsection{Learning Perceptual Features}

After the perceptual task is completed, each reference movie (for each person) is mapped to a particular synthetic movie. For each selected synthetic movie, there exists a 


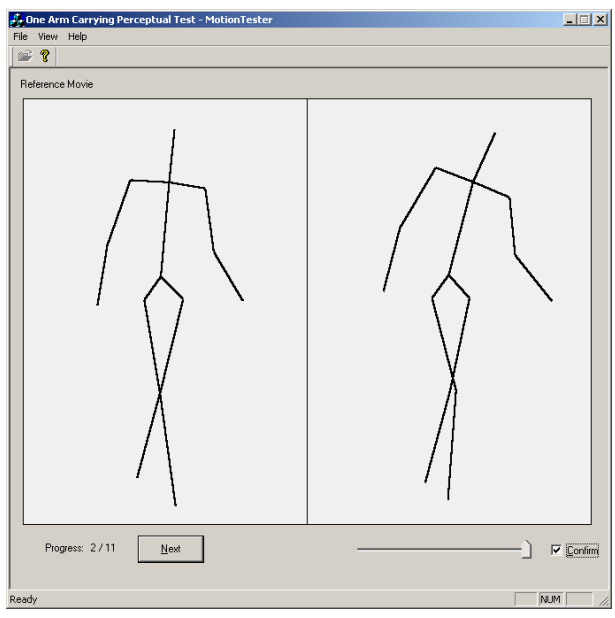

Figure 3. Screen-shot of matching program.

known $e$ value (used to generate the movie). The mean and standard deviation for the reference-synthetic $e$ mappings of the nine people are then computed (not including the illusory movies). Using the mean $e$ values, Eqn. 12 is converged to the expressive feature weights needed to bring the model estimation of effort (Eqn. 8) into alignment with the human judgements of the movements. The learning is performed using the 2-D joint-angle three-mode model of the lightest and heaviest carry examples (the position $e$ mappings are the same in this space) with $99.9 \%$ of the variance captured (the number of components in $G, H$, and $E$ were 8,8 , and 1 , respectively).

\section{Results}

We present the results of the perceptual matching task in Fig. 4.a. Rather than a smooth mapping from low (light) to high (heavy) effort, the 7 lower-effort carry movements visually appeared similar, yet distinct from the remaining 2 higher-effort carry movements. The average mapping correlation of pairwise subjects was $\rho=0.91$ (SD 0.05). The standard least-squares effort estimate is also shown for comparison. There is a noticeable difference around reference movies 6 and 7 .

The mean $e$ values determined from the perceptual mapping task were used in Eqn. 12 to learn the expressive feature weights. The method consistently converged to the same trajectory weights, with the lowerback (.36), right-hip (.15), neck (.59), and right-elbow (1.0) determined to be the expressive features (See Table 1.a). These results are meaningful as they relate to the increased leaning of the body and straightening of the carry arm as weight was added to the bag. The left counterbalance arm was not found to be an expressive feature even though it had considerable de-

\begin{tabular}{c|cccccccccc} 
& \multicolumn{11}{|c}{ Angular Expressive Weights } \\
& 1 & 2 & 3 & 4 & 5 & 6 & 7 & 8 & 9 & 10 \\
\hline (a) & .36 & 0 & 0 & .15 & 0 & .59 & 0 & 0 & 0 & 1 \\
(b) & .35 & .03 & 0 & .35 & 0 & .61 & .02 & 0 & .09 & 1
\end{tabular}

Table 1. Learned expressive weights (normalized) for the carrying examples using (a) perceptual means and (b) perceptual means with random noise trials.

viation from low to high effort (thus affecting the standard least-squares estimation). In Fig. 4.b, the efforts estimated with the learned three-mode model are compared with the perceptual means.

We additionally tested the sensitivity of the gradient descent algorithm by adding random noise to the perceptual mean values (within $.5 \mathrm{SD}$ for each reference movie). The normalized average of the expressive weights computed for 100 random-augmented mappings showed a similar result (See Table 1.b), but introduced very small weights for the left-hip (.03), left-shoulder (.02), and right-shoulder (.09).

To further demonstrate that standard least-squares estimation is not sufficient to produce perceptually-valid results for this class of motion, we turn to the illusory movements. Figure 4.c illustrates that the non-expressive least-squares estimation maps the illusory movements to basically the same effort in the angular three-mode basis. Perceptually, the result is quite different. The illusory movements were perceived to be significantly different (Mann-Whitney U test: $\mathrm{U}=81, p<0.0003)$. The results of the learned model more closely resemble the perceptual choices than do the standard least-squares results, even though the illusory data was not used to train the model. This supports our hypothesis that all motion features are not equal during motion recognition.

\section{Computational Relevance}

The recognition method provides a fast and efficient computation of action effort. Additionally, the approach shows that standard SSE computations do not produce perceptual results for the motions examined. Once the system has been trained, the recognition of action effort is computed using Eqn. 8. The $\alpha_{i j}$ values and the denominator of this equation can be computed off-line prior to recognition. Since only those feature- $i$ trajectories with non-zero expressibility weights are used to determine the effort, trajectories with zero weight can be removed from the computation, thus reducing the total amount of processed data to only the most expressive trajectories for the category. This has an added advantage in that the approach can therefore accommodate occlusions of non-expressive trajectories. 


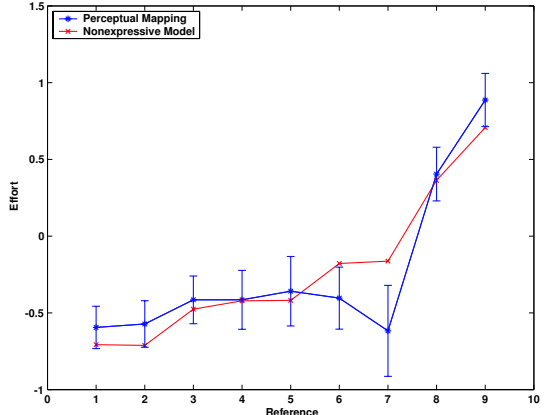

(a)

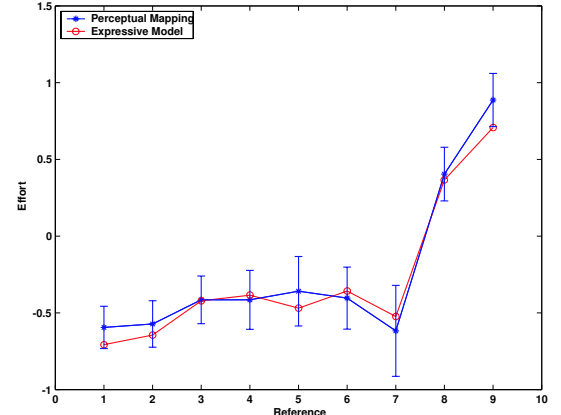

(b)

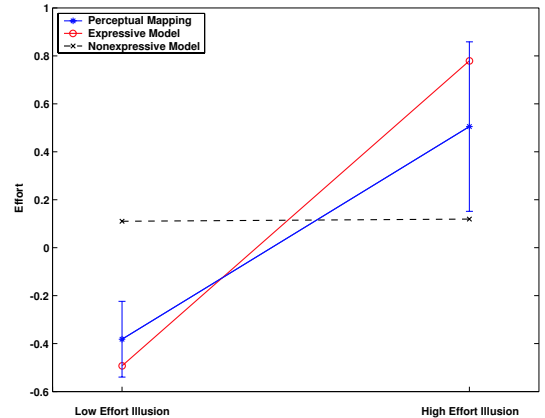

(c)

Figure 4. (a) Perceptual (mean \pm 1SD) and least-squares effort estimation for real motions. (b) Perceptual and learned efforts of real motions. (c) Perceptual, learned, and least-squares efforts of illusory movements.

\section{Conclusion}

We presented an expressive feature model for analyzing and recognizing action effort. Initially, a set of low and high effort examples for an action are factored into its three-mode principal components. Using a perceptuallybased mapping of real to model-generated synthetic movements, a weighted-SSE minimization technique learned the expressive motion trajectories needed for the model to produce effort labels similar to human judgements. The approach was demonstrated with carrying examples and two illusory movements to show the improvement of the threemode weighted-SSE technique over a non-expressive SSE approach. Future work includes investigating an automapping procedure that can mimic the human matching process, examining additional categories (walking, running, throwing, lifting, etc.), incorporating natural video input, and modeling the effort regularities across multiple people.

\section{References}

[1] J. Aggarwal and Q. Cai. Human motion analysis: a review. In Nonrigid and Articulated Motion Workshop, pages 90102. IEEE, 1997.

[2] M. Black, Y. Yacoob, A. Jepson, and D. Fleet. Learning parameterized models of image motion. In Proc. Comp. Vis. and Pattern Rec., pages 561-567, 1997.

[3] M. Brand and A. Hertzmann. Style machines. In Proc. SIGGRAPH, pages 183-192. ACM, July 2000.

[4] Y. Cui, D. Swets, and J. Weng. Learning-based hand sign recognition using SHOSLIF-M. In Proc. Int. Conf. Comp. Vis., pages 631-636. IEEE, 1995.

[5] J. Davis and V. Kannappan. Expressive features for movement exaggeration. In SIGGRAPH Conference Abstracts and Applications, page 182. ACM, 2002.
[6] D. Gavrila. The visual analysis of human movement: a survey. Computer Vision and Image Understanding, 73(1):8298, 1999.

[7] P. Kroonenberg and J. Leeuw. Principal component analysis of three-mode data by means of alternating least squares algorithms. Psychometrika, 45(1):69-97, 1980.

[8] H. Murase and S. Nayar. Visual learning and recognition of 3D objects from appearance. Int. J. of Comp. Vis., 14(1):524, 1995.

[9] R. Neal, C. Snyder, and P. Kroonenberg. Individual differences and segment interactions in throwing. Human Movement Sci., 10:653-676, 1991.

[10] W. Richards, A. Jepson, and J. Feldman. Priors, preferences and categorical percepts. In Perception as Bayesian Inference, pages 93-122. Cambridge Univ. Press, 1996.

[11] R. Rosales and S. Sclaroff. Inferring body pose without tracking body parts. In Proc. Comp. Vis. and Pattern Rec., pages 721-727. IEEE, 2000.

[12] S. Runeson and G. Frykholm. Visual perception of lifted weight. J. of Exp. Psych., 7(4):733-740, 1981.

[13] J. Tenenbaum and W. Freeman. Separating style and content. Advances in Neural Information Processing Systems, 10:662-668, 1997.

[14] L. Tucker. Some mathematical notes on three-mode factor analysis. Psychometricka, 31(3):279-311, 1966.

[15] M. Turk and A. Pentland. Eigenfaces for recognition. J. Cognitive Neuroscience, 3(1):71-86, 1991.

[16] M. Unuma, K. Anjyo, and R. Takeuchi. Fourier principles for emotion-based human figure animation. In Proc. SIGGRAPH, pages 91-96. ACM, 1995.

[17] M. Vasilescu and D. Terzopoulos. Multilinear analysis of image ensembles: TensorFaces. In Proc. European Conf. Comp. Vis., pages 447-460, 2002.

[18] M. Vasilescu. Human motion signatures for character animation. In SIGGRAPH Conference Abstracts and Applications, page 200. ACM, 2001.

[19] A. Wilson and A. Bobick. Parametric Hidden Markov Models for gesture recognition. IEEE Trans. Patt. Analy. and Mach. Intell., 21(9):884-900, 1999. 